\title{
Estimations of fractional integral operators for convex functions and related results
}

\author{
Zhihua Chen ${ }^{1}$, Ghulam Farid ${ }^{2 *}$, Atiq Ur Rehman² and Naveed Latif ${ }^{3}$
}

"Correspondence:

faridphdsms@hotmail.com;

ghlmfarid@cuiatk.edu.pk

${ }^{2}$ Department of Mathematics,

COMSATS University Islamabad,

Attock, Pakistan

Full list of author information is

available at the end of the article

\section{글 Springer}

\begin{abstract}
This research investigates the bounds of fractional integral operators containing an extended generalized Mittag-Leffler function as a kernel via several kinds of convexity. In particular, the established bounds are studied for convex functions and further connected with known results. Furthermore, these results applied to the parabolic function and consequently recurrence relations for Mittag-Leffler functions are obtained. Moreover, some fractional differential equations containing Mittag-Leffler functions are constructed and their solutions are provided by Laplace transform technique.

Keywords: Convex function; Mittag-Leffler function; Generalized fractional integral operators; Fractional differential equations
\end{abstract}

\section{Introduction}

Fractional calculus is the generalization of classical calculus. Fractional integral/derivative operators play a key role in the development of fractional calculus. They have been used to formulate various physical and dynamic problems in fractional models. The complex behavior of physical systems can be represented in terms of fractional models. For applications of fractional calculus operators in sciences and engineering we refer to reader to $[5-7,19,26,51]$. Physical properties of viscoelastic material can be interpreted by a model of fractional derivatives [4]. Furthermore, fractional calculus is applied to physics [21], bioengineering [29] optics [9, 18, 25], fluid flow [12], energy systems [11, 28] and biology [22-24].

On the other hand fractional integral/derivative operators have been used to construct and formulate new results in the theory of inequalities. Many of the well-known inequalities and related results are generalized and extended via fractional integral/derivative operators; see [2, 14-17, 30-32, 44, 52] and the references therein. At the same time convexity plays a vital role in enhancing the theory of inequalities, and facilitates optimization theory, mathematical analysis, mathematical statistics, graph theory with many other subjects. Fractional integral inequalities being suitable constraints provide existence and uniqueness of solutions for several mathematical problems in the form of fractional models.

The goal of this paper is the study of fractional integral operators containing MittagLeffler functions in their kernels. These operators are comprised in a single definition

(c) The Author(s) 2020. This article is licensed under a Creative Commons Attribution 4.0 International License, which permits use sharing, adaptation, distribution and reproduction in any medium or format, as long as you give appropriate credit to the original author(s) and the source, provide a link to the Creative Commons licence, and indicate if changes were made. The images or other third party material in this article are included in the article's Creative Commons licence, unless indicated otherwise in a credit line to the material. If material is not included in the article's Creative Commons licence and your intended use is not permitted by statutory regulation or exceeds the permitted use, you will need to obtain permission directly from the copyright holder. To view a copy of this licence, visit http://creativecommons.org/licenses/by/4.0/. 
(Eq. (2.4) and Remark 1). We will analyze them for a generalized notion of convexity called $(h-m)$-convexity. The method of proving the results of this paper can be utilized to get the results for other kinds of fractional and conformable integrals/derivatives already exist in the literature which authors will may consider for their future work; for instance for convenience Caputo-Fabrizio derivatives $[3,10]$ can be used.

\section{Preliminary results}

In this section we give definitions and notions which will be useful to establish the results of this paper.

Definition 1 Let $I$ be an interval in $\mathbb{R}$. A function $f: I \rightarrow \mathbb{R}$ is said to be convex if, for all $a, b \in I$ and $0 \leq t \leq 1$, the following inequality holds:

$$
f(t a+(1-t) b) \leq t f(a)+(1-t) f(b) .
$$

Convex functions are further generalized in different ways. One of the generalizations of convex functions is called $(h-m)$-convexity that contains several kinds of convexity for example $h$-convexity, $m$-convexity, $s$-convexity defined on the right half of real line including zero (see $[35,45])$.

Definition 2 Let $J \subseteq \mathbb{R}$ be an interval containing $(0,1)$ and let $h: J \rightarrow \mathbb{R}$ be a non-negative function. A function $f:[0, b] \rightarrow \mathbb{R}$ is called $(h-m)$-convex function, if $f$ is non-negative and for all $x, y \in[0, b], m \in[0,1]$ and $\alpha \in(0,1)$, one has

$$
f(\alpha x+m(1-\alpha) y) \leq h(\alpha) f(x)+m h(1-\alpha) f(y) .
$$

In the solution of integral and differential equations, the exponential function arises while in the solutions of fractional integral and differential equations, Mittag-Leffler function appears naturally. The Mittag-Leffler function is defined as follows [33]:

$$
E_{\alpha}(z)=\sum_{n=0}^{\infty} \frac{z^{n}}{\Gamma(\alpha n+1)} ; \quad z \in \mathbb{C}, \alpha \in \mathbb{C} ; \Re(\alpha)>0 .
$$

The Mittag-Leffler functions are used in many areas of science and engineering, especially in the theory of fractional differential equations, in solutions of generalized fractional kinetic equations (see [40]). The Mittag-Leffler function was generalized by many mathematicians: for example Wiman [46], Agarwal [1], Prabhakar [36], Shukla and Prajapati [41], Salim [38], Salim and Faraj [39], Rahman et al. [37]. For a detailed study of this function see $[20,27,36,37,39,41-43]$.

Recently in [2], Andrić et al. defined the extended generalized Mittag-Leffler function $E_{\mu, \sigma, l}^{\gamma, \delta, k, c}(\cdot ; p)$ as follows.

Definition 3 Let $\mu, \alpha, l, \gamma, c \in \mathbb{C}, \Re(\mu), \Re(\alpha), \Re(l)>0, \Re(c)>\Re(\gamma)>0$ with $p \geq 0, \delta>0$ and $0<k \leq \delta+\Re(\mu)$. Then the extended generalized Mittag-Leffler function $E_{\mu, \alpha, l}^{\gamma, \delta, c}(t ; p)$ is defined by

$$
E_{\mu, \alpha, l}^{\gamma, \delta, k, c}(t ; p)=\sum_{n=0}^{\infty} \frac{\beta_{p}(\gamma+n k, c-\gamma)}{\beta(\gamma, c-\gamma)} \frac{(c)_{n k}}{\Gamma(\mu n+\alpha)} \frac{t^{n}}{(l)_{n \delta}},
$$


where $\beta_{p}$ is the generalized beta function defined by

$$
\beta_{p}(x, y)=\int_{0}^{1} t^{x-1}(1-t)^{y-1} e^{-\frac{p}{t(1-t)}} d t
$$

and $(c)_{n k}$ is the Pochhammer symbol, $(c)_{n k}=\frac{\Gamma(c+n k)}{\Gamma(c)}$.

Lemma 1 ([2]) If $m \in N, \omega, \mu, \alpha, l, \gamma, c \in \mathbb{C}, \Re(\mu), \Re(\alpha), \Re(l)>0, \Re(c)>\Re(\gamma)>0$ with $p \geq$ $0, \delta>0$ and $0<k<\delta+\Re(\mu)$, then

$$
\left(\frac{d}{d t}\right)^{m}\left[t^{\alpha-1} E_{\mu, \alpha, l}^{\gamma, \delta, k, c}\left(\omega t^{\mu} ; p\right)\right]=t^{\alpha-m-1} E_{\mu, \alpha-m, l}^{\gamma, \delta, c, c}\left(\omega t^{\mu} ; p\right) ; \quad \Re(\alpha)>m .
$$

The left and right sided fractional integral operators corresponding to the Mittag-Leffler function (2.2) are defined as follows.

Definition 4 ([2]) Let $\omega, \mu, \alpha, l, \gamma, c \in \mathbb{C}, \Re(\mu), \Re(\alpha), \Re(l)>0, \Re(c)>\Re(\gamma)>0$ with $p \geq 0$, $\delta>0$ and $0<k \leq \delta+\Re(\mu)$. Let $f \in L_{1}[a, b]$ and $x \in[a, b]$. Then the generalized fractional integral operators $\epsilon_{\mu, \alpha, l, \omega, a^{+}}^{\gamma, \delta, c} f$ and $\epsilon_{\mu, \alpha, l, \omega, b^{-}}^{\gamma, \delta, k} f$ are defined by

$$
\begin{aligned}
& \left(\epsilon_{\mu, \alpha, l, \omega, a}^{\gamma, \delta, k, c} f\right)(x ; p)=\int_{a}^{x}(x-t)^{\alpha-1} E_{\mu, \alpha, l}^{\gamma, \delta, k, c}\left(\omega(x-t)^{\mu} ; p\right) f(t) d t, \\
& \left(\epsilon_{\mu, \alpha, l, l, \omega, b}^{\gamma, \delta, k, c} f\right)(x ; p)=\int_{x}^{b}(t-x)^{\alpha-1} E_{\mu, \alpha, l}^{\gamma, \delta, k, c}\left(\omega(t-x)^{\mu} ; p\right) f(t) d t .
\end{aligned}
$$

The following remark provides the connection of integral operators with already known fractional integral operators.

Remark 1 The operator in (2.4) contains various fractional operators:

(i) Setting $p=0$, it reduces to the fractional integral operator defined by Salim-Faraj in [39].

(ii) Setting $l=\delta=1$, it reduces to the fractional integral operator defined by Rahman et al. in [37].

(iii) Setting $p=0$ and $l=\delta=1$, it reduces to the fractional integral operator defined by Srivastava and Tomovski in [42].

(iv) Setting $p=0$ and $l=\delta=k=1$, it reduces to the fractional integral operator defined by Prabhakar in [36].

(v) Setting $p=\omega=0$, it reduces to the Riemann-Liouville fractional integral.

Fractional integral/derivative operators containing Mittag-Leffler functions are studied extensively with the prospect of their utilization in different fields; see $[8,27,36-38,41$, 42].

The following formulas are frequently used [16] in this paper:

$$
\begin{aligned}
& \left(\epsilon_{\mu, \alpha, l, c, a^{+}}^{\gamma, \delta, k, c} 1\right)(x ; p)=(x-a)^{\alpha} E_{\mu, \alpha+1, l}^{\gamma, \delta, k, c}\left(\omega(x-a)^{\mu} ; p\right):=C_{\alpha, a^{+}}(x ; p), \\
& \left(\epsilon_{\mu, \beta, \beta, l, c, b^{-}}^{\gamma, \delta, c} 1\right)(x ; p)=(b-x)^{\beta} E_{\mu, \beta+1, l}^{\gamma, \delta, k, c}\left(\omega(b-x)^{\mu} ; p\right):=C_{\beta, b^{-}}(x ; p) .
\end{aligned}
$$

The following lemmas are useful in establishing Hadamard type estimations. 
Lemma 2 ([14]) Let $f:[a, b] \longrightarrow \mathbb{R}$ be a convex function. Iff is symmetric about $\frac{a+b}{2}$, then the following inequality holds:

$$
f\left(\frac{a+b}{2}\right) \leq f(x), \quad x \in[a, b] .
$$

Lemma 3 ([15]) Let $f:[0, \infty) \rightarrow \mathbb{R}$ be $a(h-m)$-convex function. If $0 \leq a<b$ and $f(x)=$ $f\left(\frac{a+b-x}{m}\right)$, then the following inequality holds:

$$
f\left(\frac{a+b}{2}\right) \leq(m+1) h\left(\frac{1}{2}\right) f(x), \quad x \in[a, b] .
$$

The rest of the paper is organized as follows: In Sect. 2 the bounds of sum of the left and right sided generalized fractional integral operators (2.4) and (2.5) via $(h-m)$-convex functions are established. These bounds hold for several kinds of convexity as well as for several fractional integral operators. Further in Sect. 3 they are computed for the parabolic function $y=x^{2}$, and as a result recurrence relations for Mittag-Leffler functions are obtained. Section 4 consists of generalized fractional differential equations and their solutions are computed in terms of the Mittag-Leffler function.

\section{Bounds of generalized fractional integral operators}

Theorem 1 Let $f:[a, b] \rightarrow \mathbb{R}, 0 \leq a<b$, be a real valued function. If $f$ is positive and $(h-m)$-convex, then, for $\alpha, \beta \geq 1$, we have

$$
\begin{aligned}
& \left(\epsilon_{\mu, \alpha, l, \omega, a^{+}}^{\gamma, \delta, k, c} f\right)(x ; p)+\left(\epsilon_{\mu, \beta, l, \omega, b}^{\gamma, \delta, k, c} f\right)(x ; p) \\
& \leq\left((x-a) f(a) C_{\alpha-1, a^{+}}(x ; p)+(b-x) f(b) C_{\beta-1, b^{-}}(x ; p)\right. \\
& \left.\quad+m f\left(\frac{x}{m}\right)\left((x-a) C_{\alpha-1, a^{+}}(x ; p)+(b-x) C_{\beta-1, b^{-}}(x ; p)\right)\right) \int_{0}^{1} h(z) d z
\end{aligned}
$$

Proof Let $x \in[a, b]$. Then first we observe the function $f$ on the interval $[a, x]$; for $t \in[a, x]$ and $\alpha \geq 1$, one has the following inequality:

$$
(x-t)^{\alpha-1} E_{\mu, \alpha, l}^{\gamma, \delta, k, c}\left(\omega(x-t)^{\mu} ; p\right) \leq(x-a)^{\alpha-1} E_{\mu, \alpha, l}^{\gamma, \delta, k, c}\left(\omega(x-a)^{\mu} ; p\right) .
$$

As $f$ is $(h-m)$-convex, so for $t \in[a, x]$, we have

$$
f(t) \leq h\left(\frac{x-t}{x-a}\right) f(a)+m h\left(\frac{t-a}{x-a}\right) f\left(\frac{x}{m}\right) .
$$

Multiplying (3.2) and (3.3), then integrating over $[a, x]$, we get

$$
\begin{aligned}
& \int_{a}^{x}(x-t)^{\alpha-1} E_{\mu, \alpha, l}^{\gamma, \delta, k, c}\left(\omega(x-t)^{\mu} ; p\right) f(t) d t \\
& \leq f(a)(x-a)^{\alpha-1} E_{\mu, \alpha, l}^{\gamma, \delta, k, c}\left(\omega(x-a)^{\mu} ; p\right) \int_{a}^{x} h\left(\frac{x-t}{x-a}\right) d t \\
& \quad+m f\left(\frac{x}{m}\right)(x-a)^{\alpha-1} E_{\mu, \alpha, l}^{\gamma, \delta, k}\left(\omega(x-a)^{\mu} ; p\right) \int_{a}^{x} h\left(\frac{t-a}{x-a}\right) d t .
\end{aligned}
$$


By using (2.4) on left hand side and (2.6) on right hand side, we have

$$
\left(\epsilon_{\mu, \alpha, l, \omega, a^{+}}^{\gamma, \delta, k, c} f\right)(x ; p) \leq(x-a) C_{\alpha-1, a^{+}}(x ; p)\left(f(a)+m f\left(\frac{x}{m}\right)\right) \int_{0}^{1} h(z) d z .
$$

Now on the other hand we address the function $f$ on the interval $[x, b]$; for $t \in[x, b]$ and $\beta \geq 1$, one has the following inequality:

$$
(t-x)^{\beta-1} E_{\mu, \beta, l}^{\gamma, \delta, k, c}\left(\omega(t-x)^{\mu} ; p\right) \leq(b-x)^{\beta-1} E_{\mu, \beta, l}^{\gamma, \delta, k, c}\left(\omega(b-x)^{\mu} ; p\right) .
$$

Again from $(h-m)$-convexity of $f$ for $t \in[x, b]$, we have

$$
f(t) \leq h\left(\frac{t-x}{b-x}\right) f(b)+m h\left(\frac{b-t}{b-x}\right) f\left(\frac{x}{m}\right)
$$

Similarly multiplying (3.6) and (3.7), then integrating over $[x, b]$, we get

$$
\left(\epsilon_{\mu, \beta, l, \omega, b^{-}}^{\gamma, \delta, k, c} f\right)(x ; p) \leq(b-x) C_{\beta-1, b^{-}}(x ; p)\left(f(b)+m f\left(\frac{x}{m}\right)\right) \int_{0}^{1} h(z) d z .
$$

Adding (3.5) and (3.8), inequality (3.1) is obtained.

If $m=1$ and $h(z)=z$ in (3.1), then the following result holds for a convex function.

Corollary 1 Let $f:[a, b] \rightarrow \mathbb{R}, a<b$, be a real valued function. Iff is positive and convex, then, for $\alpha, \beta \geq 1$, we have

$$
\begin{aligned}
& \left(\epsilon_{\mu, \alpha, l, \omega, a^{+}}^{\gamma, \delta, k),} f(x ; p)+\left(\epsilon_{\mu, \beta, l, \omega, b^{-}}^{\gamma, \delta, k, c} f\right)(x ; p)\right. \\
& \leq \frac{(x-a) f(a) C_{\alpha-1, a^{+}}(x ; p)+(b-x) f(b) C_{\beta-1, b^{-}}(x ; p)}{2} \\
& \quad+f(x)\left[\frac{(x-a) C_{\alpha-1, a^{+}}(x ; p)+(b-x) C_{\beta-1, b^{-}}(x ; p)}{2}\right] .
\end{aligned}
$$

Remark 2 If $\omega=p=0$ in (3.9), then [14, Theorem 1] is obtained.

Theorem 2 Let $f:[a, b] \rightarrow \mathbb{R}, 0 \leq a<b$, be a real valued function. Iff is differentiable and $\left|f^{\prime}\right|$ is $(h-m)$-convex, then, for $\alpha, \beta \geq 1$, we have

$$
\begin{aligned}
\mid\left(\epsilon_{\mu, \alpha-1, l, \omega, a^{+}}^{\gamma, \delta, k, c} f\right)(x ; p)+\left(\epsilon_{\mu, \beta-1, l, \omega, b^{-}}^{\gamma, \delta, k, c} f\right)(x ; p) & \\
& -\left(f(a) C_{\alpha-1, a^{+}}(x ; p)+f(b) C_{\beta-1, b^{-}}(x ; p)\right) \mid \\
\leq & \left((x-a)\left|f^{\prime}(a)\right| C_{\alpha-1, a^{+}}(x ; p)+(b-x)\left|f^{\prime}(b)\right| C_{\beta-1, b^{-}}(x ; p)\right. \\
\quad & \left.+m\left|f^{\prime}\left(\frac{x}{m}\right)\right|\left((x-a) C_{\alpha-1, a^{+}}(x ; p)+(b-x) C_{\beta-1, b^{-}}(x ; p)\right)\right) \int_{0}^{1} h(z) d z .
\end{aligned}
$$

Proof Let $x \in[a, b]$ and $t \in[a, x]$. Then using $(h-m)$-convexity of $\left|f^{\prime}\right|$, we have

$$
\left|f^{\prime}(t)\right| \leq h\left(\frac{x-t}{x-a}\right)\left|f^{\prime}(a)\right|+m h\left(\frac{t-a}{x-a}\right)\left|f^{\prime}\left(\frac{x}{m}\right)\right| \text {. }
$$


From (3.11), one has

$$
f^{\prime}(t) \leq h\left(\frac{x-t}{x-a}\right)\left|f^{\prime}(a)\right|+m h\left(\frac{t-a}{x-a}\right)\left|f^{\prime}\left(\frac{x}{m}\right)\right|
$$

Multiplying (3.2) and (3.12), then integrating over $[a, x]$, we get

$$
\begin{aligned}
& \int_{a}^{x}(x-t)^{\alpha-1} E_{\mu, \alpha, l}^{\gamma, \delta, k, c}\left(\omega(x-t)^{\mu} ; p\right) f^{\prime}(t) d t \\
& \leq(x-a)^{\alpha-1} E_{\mu, \alpha, l}^{\gamma, \delta, k, c}\left(\omega(x-a)^{\mu} ; p\right) \\
& \quad \times\left[\left|f^{\prime}(a)\right| \int_{a}^{x} h\left(\frac{x-t}{x-a}\right) d t+m\left|f^{\prime}\left(\frac{x}{m}\right)\right| \int_{a}^{x} h\left(\frac{t-a}{x-a}\right) d t\right] .
\end{aligned}
$$

The left hand side is calculated thus: Put $x-t=z$, that is, $t=x-z$, also using the derivative property (2.3) of the Mittag-Leffler function, we have

$$
\begin{aligned}
& \int_{0}^{x-a} z^{\alpha-1} E_{\mu, \alpha, l}^{\gamma, \delta, k, c} 1\left(\omega z^{\mu} ; p\right) f^{\prime}(x-z) d z \\
& \quad=-(x-a)^{\alpha-1} E_{\mu, \alpha, l}^{\gamma, \delta, k, c}\left(\omega(x-a)^{\mu} ; p\right) f(a)+\int_{0}^{x-a} z^{\alpha-2} E_{\mu, \alpha-1, l}^{\gamma, \delta, k, c}\left(\omega z^{\mu} ; p\right) f(x-z) d z,
\end{aligned}
$$

now by putting $x-z=t$, in second term of the right hand side of the above equation and by using (2.4) and (2.6), we get

$$
\int_{0}^{x-a} z^{\alpha-1} E_{\mu, \alpha, l}^{\gamma, \delta, k, c}\left(\omega z^{\mu} ; p\right) f^{\prime}(x-z) d z=-f(a) C_{\alpha-1, a^{+}}(x ; p)+\left(\epsilon_{\mu, \alpha-1, l, \omega, a^{+}}^{\gamma, \delta, k, c} f\right)(x ; p) .
$$

Therefore, (3.13) takes the form

$$
\begin{aligned}
& \left(\epsilon_{\mu, \alpha-1, l, \omega, a^{+}}^{\gamma, \delta} f\right)(x ; p)-f(a) C_{\alpha-1, a^{+}}(x ; p) \\
& \quad \leq(x-a) C_{\alpha-1, a^{+}}(x ; p)\left(\left|f^{\prime}(a)\right|+m\left|f^{\prime}\left(\frac{x}{m}\right)\right|\right) \int_{0}^{1} h(z) d z .
\end{aligned}
$$

Also from (3.11), one has

$$
f^{\prime}(t) \geq-\left(h\left(\frac{x-t}{x-a}\right)\left|f^{\prime}(a)\right|+m h\left(\frac{t-a}{x-a}\right)\left|f^{\prime}\left(\frac{x}{m}\right)\right|\right) .
$$

Following the same procedure as one did for (3.12), we also have

$$
\begin{aligned}
& f(a) C_{\alpha-1, a^{+}}(x ; p)-\left(\epsilon_{\mu, \alpha-1, l, \omega, a^{+}}^{\gamma, \delta, k, c} f\right)(x ; p) \\
& \quad \leq(x-a) C_{\alpha-1, a^{+}}(x ; p)\left(\left|f^{\prime}(a)\right|+m\left|f^{\prime}\left(\frac{x}{m}\right)\right|\right) \int_{0}^{1} h(z) d z .
\end{aligned}
$$

From (3.14) and (3.16), we get

$$
\begin{aligned}
& \left|\left(\epsilon_{\mu, \alpha-1, l, \omega, a^{+}}^{\gamma, \delta, k, c}\right)(x ; p)-f(a) C_{\alpha-1, a^{+}}(x ; p)\right| \\
& \quad \leq(x-a) C_{\alpha-1, a^{+}}(x ; p)\left(\left|f^{\prime}(a)\right|+m\left|f^{\prime}\left(\frac{x}{m}\right)\right|\right) \int_{0}^{1} h(z) d z .
\end{aligned}
$$


Now let $t \in[x, b]$. Then using $(h-m)$-convexity of $\left|f^{\prime}\right|$, we have

$$
\left|f^{\prime}(t)\right| \leq h\left(\frac{t-x}{b-x}\right)\left|f^{\prime}(b)\right|+m h\left(\frac{b-t}{b-x}\right)\left|f^{\prime}\left(\frac{x}{m}\right)\right| \text {. }
$$

Along the same lines as for (3.2), (3.12) and (3.15), one can get from (3.6) and (3.18) the following inequality:

$$
\begin{aligned}
& \left|\left(\epsilon_{\mu, \beta-1, l, \omega, b^{-}}^{\gamma, \delta, k, c}\right)(x ; p)-f(b) C_{\beta-1, b^{-}}(x ; p)\right| \\
& \quad \leq(b-x) C_{\beta-1, b^{-}}(x ; p)\left(\left|f^{\prime}(b)\right|+m\left|f^{\prime}\left(\frac{x}{m}\right)\right|\right) \int_{0}^{1} h(z) d z .
\end{aligned}
$$

From (3.17) and (3.19) via the triangular inequality, inequality (3.10) is obtained.

If $m=1$ and $h(z)=z$ in (3.10), then the following result holds for a convex function.

Corollary 2 Let $f:[a, b] \longrightarrow \mathbb{R}, a<b$, be a real valued function. If $f$ is differentiable and $\left|f^{\prime}\right|$ is convex, then, for $\alpha, \beta \geq 1$, we have

$$
\begin{aligned}
\mid\left(\epsilon_{\mu, \alpha-1, l, \omega, a^{+}}^{\gamma, \delta, k, c} f\right)(x ; p)+\left(\epsilon_{\mu, \beta-1, l, \omega, b^{-}}^{\gamma, \delta, k, c} f\right)(x ; p) \\
\quad-\left(f(a) C_{\alpha-1, a^{+}}(x ; p)+f(b) C_{\beta-1, b^{-}}(x ; p)\right) \mid \\
\leq \frac{(x-a)\left|f^{\prime}(a)\right| C_{\alpha-1, a^{+}}(x ; p)+(b-x)\left|f^{\prime}(b)\right| C_{\beta-1, b^{-}}(x ; p)}{2} \\
\quad+\left|f^{\prime}(x)\right|\left(\frac{(x-a) C_{\alpha-1, a^{+}}(x ; p)+(b-x) C_{\beta-1, b^{-}}(x ; p)}{2}\right) .
\end{aligned}
$$

\section{Remark 3}

(i) If $\omega=p=0$ and replace $\alpha$ by $\alpha+1$ in (3.20), then [14, Theorem 2] is obtained.

(ii) If $\omega=p=0, \alpha=\beta=1$ and $f^{\prime}$ passes through $x=\frac{a+b}{2}$, then from (3.20) [13, Theorem 2.2] is obtained.

Theorem 3 Let $f:[a, b] \rightarrow \mathbb{R}, 0 \leq a<b$, be a real valued function. Iff is positive, $(h-m)$ convex and $f(x)=f\left(\frac{a+b-x}{m}\right)$, then, for $\alpha, \beta>0$, we have

$$
\begin{aligned}
& \frac{f\left(\frac{a+b}{2}\right)}{(m+1) h\left(\frac{1}{2}\right)}\left[C_{\beta+1, b^{-}}(a ; p)+C_{\alpha+1, a^{+}}(b ; p)\right] \\
& \quad \leq\left(\epsilon_{\mu, \beta+1, l, \omega, b^{-}}^{\gamma, \delta, k, c}\right)(a ; p)+\left(\epsilon_{\mu, \alpha+1, l, \omega, a^{+}}^{\gamma, \delta, c} f\right)(b ; p) \\
& \quad \leq(b-a)^{2}\left[C_{\beta-1, b^{-}}(a ; p)+C_{\alpha-1, a^{+}}(b ; p)\right]\left(f(a)+m f\left(\frac{b}{m}\right)\right) \int_{0}^{1} h(z) d z .
\end{aligned}
$$

Proof For $x \in[a, b]$, we have

$$
(x-a)^{\beta} E_{\mu, \beta, l}^{\gamma, \delta, k, c}\left(\omega(x-a)^{\mu} ; p\right) \leq(b-a)^{\beta} E_{\mu, \beta, l}^{\gamma, \delta, k, c}\left(\omega(b-a)^{\mu} ; p\right), \quad \beta>0 .
$$

As $f$ is $(h-m)$-convex, for $x \in[a, b]$ we have

$$
f(x) \leq m h\left(\frac{x-a}{b-a}\right) f\left(\frac{b}{m}\right)+h\left(\frac{b-x}{b-a}\right) f(a) .
$$


Multiplying (3.22) and (3.23), then integrating over $[a, b]$, we get

$$
\begin{aligned}
& \int_{a}^{b}(x-a)^{\beta} E_{\mu, \beta, l}^{\gamma, \delta, k, c}\left(\omega(x-a)^{\mu} ; p\right) f(x) d x \\
& \leq m(b-a)^{\beta} E_{\mu, \beta, l}^{\gamma, \delta, k, c}\left(\omega(b-a)^{\mu} ; p\right) f\left(\frac{b}{m}\right) \int_{a}^{b} h\left(\frac{x-a}{b-a}\right) d x \\
& \quad+(b-a)^{\beta} E_{\mu, \beta, l}^{\gamma, \delta, k, c}\left(\omega(b-a)^{\mu} ; p\right) f(a) \int_{a}^{b} h\left(\frac{b-x}{b-a}\right) d x .
\end{aligned}
$$

From this we have

$$
\left(\epsilon_{\mu, \beta+1, l, \omega, b^{-}}^{\gamma, \delta, k) c}(a ; p) \leq(b-a)^{2} C_{\beta-1, b^{-}}(a ; p)\left(f(a)+m f\left(\frac{b}{m}\right)\right) \int_{0}^{1} h(z) d z\right.
$$

On the other hand for $x \in[a, b]$, we have

$$
(b-x)^{\alpha} E_{\mu, \alpha, l}^{\gamma, \delta, k, c}\left(\omega(b-x)^{\mu} ; p\right) \leq(b-a)^{\alpha} E_{\mu, \alpha, l}^{\gamma, \delta, k, c}\left(\omega(b-a)^{\mu} ; p\right), \quad \alpha>0 .
$$

Similarly multiplying (3.23) and (3.25), then integrating over $[a, b]$, we get

$$
\left(\epsilon_{\mu, \alpha+1, l, \omega, a^{+}}^{\gamma, \delta, k, c} f\right)(b ; p) \leq(b-a)^{2} C_{\alpha-1, a^{+}}(b ; p)\left(f(a)+m f\left(\frac{b}{m}\right)\right) \int_{0}^{1} h(z) d z .
$$

Adding (3.24) and (3.26), we get

$$
\begin{aligned}
& \left(\epsilon_{\mu, \beta+1, l, \omega, b^{-}}^{\gamma, \delta, k, c} f\right)(a ; p)+\left(\epsilon_{\mu, \alpha+1, l, \omega, a^{+}}^{\gamma, \delta, k, c} f\right)(b ; p) \\
& \quad \leq(b-a)^{2}\left(C_{\beta-1, b^{-}}(a ; p)+C_{\alpha-1, a^{+}}(b ; p)\right)\left(f(a)+m f\left(\frac{b}{m}\right)\right) \int_{0}^{1} h(z) d z .
\end{aligned}
$$

Multiplying (2.9) with $(x-a)^{\beta} E_{\mu, \beta, l}^{\gamma, \delta, k, c}\left(\omega(x-a)^{\mu} ; p\right)$, then integrating over $[a, b]$, we get

$$
\begin{aligned}
& f\left(\frac{a+b}{2}\right) \int_{a}^{b}(x-a)^{\beta} E_{\mu, \beta, l}^{\gamma, \delta, k, c}\left(\omega(x-a)^{\mu} ; p\right) d x \\
& \quad \leq(m+1) h\left(\frac{1}{2}\right) \int_{a}^{b}(x-a)^{\beta} E_{\mu, \beta, l}^{\gamma, \delta, k}\left(\omega(x-a)^{\mu} ; p\right) f(x) d x
\end{aligned}
$$

by using (2.5) and (2.7), we get

$$
\frac{f\left(\frac{a+b}{2}\right)}{(m+1) h\left(\frac{1}{2}\right)} C_{\beta+1, b^{-}}(a ; p) \leq\left(\epsilon_{\mu, \beta+1, l, \omega, b^{-}}^{\gamma, \delta, k} f\right)(a ; p) .
$$

Similarly multiplying (2.9) with $(b-x)^{\alpha} E_{\mu, \alpha, l}^{\gamma, \delta, k, c}\left(\omega(b-x)^{\mu} ; p\right)$, then integrating over $[a, b]$ and using (2.4) and (2.6), one can get

$$
\frac{f\left(\frac{a+b}{2}\right)}{(m+1) h\left(\frac{1}{2}\right)} C_{\alpha+1, a^{+}}(b ; p) \leq\left(\epsilon_{\mu, \alpha+1, l, \omega, a^{+}}^{\gamma, k, c} f\right)(b ; p) .
$$


Adding (3.28) and (3.29), we get

$$
\begin{aligned}
& \frac{f\left(\frac{a+b}{2}\right)}{(m+1) h\left(\frac{1}{2}\right)}\left[C_{\beta+1, b^{-}}(a ; p)+C_{\alpha+1, a^{+}}(b ; p)\right] \\
& \quad \leq\left(\epsilon_{\mu, \beta+1, l, \omega, b^{-}}^{\gamma, \delta, k, c} f\right)(a ; p)+\left(\epsilon_{\mu, \alpha+1, l, \omega, a^{+}}^{\gamma, \delta, k, c} f\right)(b ; p) .
\end{aligned}
$$

From inequalities (3.27) and (3.30), inequality (3.21) is obtained.

If $m=1$ and $h(z)=z$ in (3.21), then the following result holds for a convex function.

Corollary 3 Let $f:[a, b] \longrightarrow \mathbb{R}, a<b$, be a real valued function. Iff is positive, convex and symmetric about $\frac{a+b}{2}$, then, for $\alpha, \beta>0$, we have

$$
\begin{aligned}
& f\left(\frac{a+b}{2}\right)\left[C_{\beta+1, b^{-}}(a ; p)+C_{\alpha+1, a^{+}}(b ; p)\right] \\
& \quad \leq\left(\epsilon_{\mu, \beta+1, l, \omega, b^{-}}^{\gamma, \delta, k, c}\right)(a ; p)+\left(\epsilon_{\mu, \alpha+1, l, \omega, a^{+}}^{\gamma, \delta, k, c}\right)(b ; p) \\
& \quad \leq(b-a)^{2}\left[C_{\beta-1, b^{-}}(a ; p)+C_{\alpha-1, a^{+}}(b ; p)\right]\left[\frac{f(a)+f(b)}{2}\right] .
\end{aligned}
$$

\section{Remark 4}

(i) If $\omega=p=0$ in (3.31), then [14, Theorem 3] is obtained.

(ii) If $\alpha=\beta \rightarrow 0$ and $\omega=p=0$, then from the above inequality, we get the Hadamard inequality.

\section{Inequalities for the extended generalized Mittag-Leffler functions}

In this section, results of previous section are applied for the function $f(x)=x^{2}$. The function $f$ is convex and $\left|f^{\prime}(x)\right|=2|x|$ is also convex. By virtue of this function we succeeded to establish recurrence relations among Mittag-Leffler functions which may be useful in the solutions of fractional boundary value problems and fractional differential equations. Ullah et al. computed generalized fractional integral operators for the function $f(x)=x^{2}$, in [44], as follows:

$$
\begin{aligned}
\left(\epsilon_{\mu, \alpha, l, \omega, a^{+}}^{\gamma, \delta, k, c} f\right)(x ; p)= & (x-a)^{\alpha}\left[a^{2} E_{\mu, \alpha+1, l}^{\gamma, \delta, k, c}\left(\omega(x-a)^{\mu} ; p\right)\right. \\
& +2 a(x-a) E_{\mu, \alpha+2, l}^{\gamma, \delta, k, c}\left(\omega(x-a)^{\mu} ; p\right) \\
& \left.+2(x-a)^{2} E_{\mu, \alpha+3, l}^{\gamma, \delta, k, c}\left(\omega(x-a)^{\mu} ; p\right)\right], \\
\left(\epsilon_{\mu, \alpha, l, \omega, b^{-}}^{\gamma, \delta, k, c} f\right)(x ; p)= & (b-x)^{\alpha}\left[b^{2} E_{\mu, \alpha+1, l}^{\gamma, \delta, k, c}\left(\omega(b-x)^{\mu} ; p\right)\right. \\
& -2 b(b-x) E_{\mu, \alpha+2, l}^{\gamma, \delta, k}\left(\omega(b-x)^{\mu} ; p\right) \\
& \left.+2(b-x)^{2} E_{\mu, \alpha+3, l}^{\gamma, \delta, k, c}\left(\omega(b-x)^{\mu} ; p\right)\right] .
\end{aligned}
$$

Below, the results of Sect. 2 are applied to obtain recurrence inequalities for Mittag-Leffler functions. 
Theorem 4 Mittag-Leffler functions satisfy the following recurrence relation:

$$
\begin{aligned}
& \frac{\left(a^{2}+b^{2}\right)}{(b-a)^{2}} E_{\mu, \alpha+1, l}^{\gamma, \delta, k}\left(\omega^{\prime}(b-a)^{\mu} ; p\right)+E_{\mu, \alpha+3, l}^{\gamma, \delta, k}\left(\omega^{\prime}(b-a)^{\mu} ; p\right) \\
& \quad \leq \frac{\left((2 m+1)\left(a^{2}+b^{2}\right)+2 a b\right)}{2 m(b-a)^{2}} E_{\mu, \alpha, l}^{\gamma, \delta, k, c}\left(\omega^{\prime}(b-a)^{\mu} ; p\right) \int_{0}^{1} h(z) d z \\
& \quad+E_{\mu, \alpha+2, l}^{\gamma, \delta, k, c}\left(\omega^{\prime}(b-a)^{\mu} ; p\right),
\end{aligned}
$$

where $\omega^{\prime}=\frac{\omega}{2^{\mu}}$.

Proof By using (4.1), (4.2) and $f(x)=x^{2}$ in (3.1) of Theorem 1, we have

$$
\begin{aligned}
(x-a)^{\alpha} & {\left[a^{2} E_{\mu, \alpha+1, l}^{\gamma, \delta, k, c}\left(\omega(x-a)^{\mu} ; p\right)+2 a(x-a) E_{\mu, \alpha+2, l}^{\gamma, \delta, c}\left(\omega(x-a)^{\mu} ; p\right)\right.} \\
& \left.+2(x-a)^{2} E_{\mu, \alpha+3, l}^{\gamma, \delta, k, c}\left(\omega(x-a)^{\mu} ; p\right)\right]+(b-x)^{\beta}\left[b^{2} E_{\mu, \beta+1, l}^{\gamma, \delta, k, c}\left(\omega(b-x)^{\mu} ; p\right)\right. \\
& \left.-2 b(b-x) E_{\mu, \beta+2, l}^{\gamma, \delta, c}\left(\omega(b-x)^{\mu} ; p\right)+2(b-x)^{2} E_{\mu, \beta+3, l}^{\gamma, \delta, k, c}\left(\omega(b-x)^{\mu} ; p\right)\right] \\
\leq & \left(\left(a^{2}+\frac{x^{2}}{m}\right)(x-a)^{\alpha} E_{\mu, \alpha, l}^{\gamma, \delta, k, c}\left(\omega(x-a)^{\mu} ; p\right)\right. \\
& \left.+\left(b^{2}+\frac{x^{2}}{m}\right)(b-x)^{\beta} E_{\mu, \beta, l}^{\gamma, \delta, k, c}\left(\omega(b-x)^{\mu} ; p\right)\right) \int_{0}^{1} h(z) d z .
\end{aligned}
$$

Now by putting $x=\frac{a+b}{2}$ and $\alpha=\beta$ in (4.4), then after simplification, inequality (4.3) is obtained.

Corollary 4 If $m=1$ and $h(z)=z$ in (4.3), then we have

$$
\begin{aligned}
& \frac{\left(a^{2}+b^{2}\right)}{(b-a)^{2}} E_{\mu, \alpha+1, l}^{\gamma, \delta, k}\left(\omega^{\prime}(b-a)^{\mu} ; p\right)+E_{\mu, \alpha+3, l}^{\gamma, \delta, k}\left(\omega^{\prime}(b-a)^{\mu} ; p\right) \\
& \quad \leq \frac{\left(3 a^{2}+3 b^{2}+2 a b\right)}{4(b-a)^{2}} E_{\mu, \alpha, l}^{\gamma, \delta, k, c}\left(\omega^{\prime}(b-a)^{\mu} ; p\right)+E_{\mu, \alpha+2, l}^{\gamma, \delta, c}\left(\omega^{\prime}(b-a)^{\mu} ; p\right) .
\end{aligned}
$$

Theorem 5 Mittag-Leffler functions satisfy the following recurrence relation:

$$
\begin{aligned}
& \left|E_{\mu, \alpha+2, l}^{\gamma, \delta, k, c}\left(\omega^{\prime}(b-a)^{\mu} ; p\right)-E_{\mu, \alpha+1, l}^{\gamma, \delta, k, c}\left(\omega^{\prime}(b-a)^{\mu} ; p\right)\right| \\
& \quad \leq \frac{1}{m(b-a)}(m a+m b+(a+b)) E_{\mu, \alpha, l}^{\gamma, \delta, k}\left(\omega^{\prime}(b-a)^{\mu} ; p\right) \int_{0}^{1} h(z) d z,
\end{aligned}
$$

where $\omega^{\prime}=\frac{\omega}{2^{\mu}}$.

Proof By using (4.1), (4.2) and $\left|f^{\prime}(x)\right|=2|x|$ in (3.10) of Theorem 2, we have

$$
\begin{aligned}
\mid(x-a)^{\alpha} & {\left[a^{2}(x-a)^{-1} E_{\mu, \alpha, l}^{\gamma, \delta, k, c}\left(\omega(x-a)^{\mu} ; p\right)+2 a E_{\mu, \alpha+1, l}^{\gamma, \delta, k, c}\left(\omega(x-a)^{\mu} ; p\right)\right.} \\
& \left.+2(x-a) E_{\mu, \alpha+2, l}^{\gamma, \delta, k, c}\left(\omega(x-a)^{\mu} ; p\right)\right]+(b-x)^{\beta}\left[b^{2}(b-x)^{-1} E_{\mu, \beta, l}^{\gamma, \delta, k}\left(\omega(b-x)^{\mu} ; p\right)\right. \\
& \left.-2 b E_{\mu, \beta+1, l}^{\gamma, \delta, c}\left(\omega(b-x)^{\mu} ; p\right)+2(b-x) E_{\mu, \beta+2, l}^{\gamma, \delta, k, c}\left(\omega(b-x)^{\mu} ; p\right)\right] \\
& -\left(a^{2}(x-a)^{\alpha-1} E_{\mu, \alpha, l}^{\gamma, \delta, k, c}\left(\omega(x-a)^{\mu} ; p\right)+b^{2}(b-x)^{\beta-1} E_{\mu, \beta, l}^{\gamma, \delta, k, c}\left(\omega(b-x)^{\mu} ; p\right)\right) \mid
\end{aligned}
$$




$$
\begin{aligned}
\leq & \left(2\left(|a|+\frac{|x|}{m}\right)(x-a)^{\alpha} E_{\mu, \alpha, l}^{\gamma, \delta, k, c}\left(\omega(x-a)^{\mu} ; p\right)\right. \\
& \left.+2\left(|b|+\frac{|x|}{m}\right)(b-x)^{\beta} E_{\mu, \beta, l}^{\gamma, \delta, k, c}\left(\omega(b-x)^{\mu} ; p\right)\right) \int_{0}^{1} h(z) d z .
\end{aligned}
$$

Now by putting $x=\frac{a+b}{2}$ and $\alpha=\beta$ in (4.7), then after simplification, inequality (4.6) is obtained.

Corollary 5 If $m=1$ and $h(z)=z$ in (4.6), then we have

$$
\begin{aligned}
& \left|E_{\mu, \alpha+2, l}^{\gamma, \delta, k, c}\left(\omega^{\prime}(b-a)^{\mu} ; p\right)-E_{\mu, \alpha+1, l}^{\gamma, \delta, k, c}\left(\omega^{\prime}(b-a)^{\mu} ; p\right)\right| \\
& \quad \leq \frac{1}{b-a}(a+b) E_{\mu, \alpha, l}^{\gamma, \delta, k, c}\left(\omega^{\prime}(b-a)^{\mu} ; p\right) .
\end{aligned}
$$

Theorem 6 Mittag-Leffler functions satisfy the following recurrence relation:

$$
\begin{gathered}
E_{\mu, \alpha+1, l}^{\gamma, \delta, k, c}\left(\omega(b-a)^{\mu} ; p\right)-\left(1+\frac{1}{m}\right) E_{\mu, \alpha, l}^{\gamma, \delta, k, c}\left(\omega(b-a)^{\mu} ; p\right) \int_{0}^{1} h(z) d z \\
\quad \leq \frac{2(b-a)^{2}}{\left(a^{2}+b^{2}\right)}\left(E_{\mu, \alpha+2, l}^{\gamma, \delta, k, c}\left(\omega(b-a)^{\mu} ; p\right)-2 E_{\mu, \alpha+3, l}^{\gamma, \delta, k, c}\left(\omega(b-a)^{\mu} ; p\right)\right) .
\end{gathered}
$$

Proof In (4.4), putting $x=a$ and $x=b$, then adding for $\alpha=\beta$, inequality (4.9) is obtained.

Corollary 6 If $m=1$ and $h(z)=z$ in (4.9), then we have

$$
\begin{aligned}
& E_{\mu, \alpha+1, l}^{\gamma, \delta, k, c}\left(\omega(b-a)^{\mu} ; p\right)-E_{\mu, \alpha, l}^{\gamma, \delta, k, c}\left(\omega(b-a)^{\mu} ; p\right) \\
& \quad \leq \frac{2(b-a)^{2}}{\left(a^{2}+b^{2}\right)}\left(E_{\mu, \alpha+2, l}^{\gamma, \delta, k}\left(\omega(b-a)^{\mu} ; p\right)-2 E_{\mu, \alpha+3, l}^{\gamma, \delta, c, c}\left(\omega(b-a)^{\mu} ; p\right)\right) .
\end{aligned}
$$

Theorem 7 Mittag-Leffler functions satisfy the following recurrence relation:

$$
\begin{aligned}
& \left|E_{\mu, \alpha+2, l}^{\gamma, \delta, k, c}\left(\omega(b-a)^{\mu} ; p\right)-\frac{1}{2} E_{\mu, \alpha+1, l}^{\gamma, \delta, k, c}\left(\omega(b-a)^{\mu} ; p\right)\right| \\
& \quad \leq \frac{\left(1+\frac{1}{m}\right)}{2(b-a)}(a+b) E_{\mu, \alpha, l}^{\gamma, \delta, k, c}\left(\omega(b-a)^{\mu} ; p\right) \int_{0}^{1} h(z) d z .
\end{aligned}
$$

Proof In (4.7), putting $x=a$ and $x=b$, then adding for $\alpha=\beta$, inequality (4.11) is obtained.

Corollary 7 If $m=1$ and $h(z)=z$ in (4.11), then we have

$$
\begin{aligned}
& \left|E_{\mu, \alpha+2, l}^{\gamma, \delta, k, c}\left(\omega(b-a)^{\mu} ; p\right)-\frac{1}{2} E_{\mu, \alpha+1, l}^{\gamma, \delta, k, c}\left(\omega(b-a)^{\mu} ; p\right)\right| \\
& \quad \leq \frac{1}{2(b-a)}(a+b) E_{\mu, \alpha, l}^{\gamma, \delta, k, c}\left(\omega(b-a)^{\mu} ; p\right) .
\end{aligned}
$$


Theorem 8 Mittag-Leffler functions satisfy the following recurrence relation:

$$
\begin{aligned}
& E_{\mu, \alpha+1, l}^{\gamma, \delta, k, c}(\omega ; p)-\left(1+\frac{1}{m}\right) E_{\mu, \alpha, l}^{\gamma, \delta, k, c}(\omega ; p) \int_{0}^{1} h(z) d z \\
& \quad \leq 2\left(E_{\mu, \alpha+2, l}^{\gamma, \delta, k, c}(\omega ; p)-2 E_{\mu, \alpha+3, l}^{\gamma, \delta, k, c}(\omega ; p)\right) .
\end{aligned}
$$

Proof In (4.9), putting $a=0$ and $b=1$, then inequality (4.13) is obtained.

Corollary 8 If $m=1$ and $h(z)=z$ in (4.13), then we have

$$
\begin{aligned}
& E_{\mu, \alpha+1, l}^{\gamma, \delta, k, c}(\omega ; p)-E_{\mu, \alpha, l}^{\gamma, \delta, k, c}(\omega ; p) \\
& \quad \leq 2\left(E_{\mu, \alpha+2, l}^{\gamma, \delta, k, c}(\omega ; p)-2 E_{\mu, \alpha+3, l}^{\gamma, \delta, k, c}(\omega ; p)\right) .
\end{aligned}
$$

Theorem 9 Mittag-Leffler functions satisfy the following recurrence relation:

$$
\begin{aligned}
& \left|2 E_{\mu, \alpha+2, l}^{\gamma, \delta, k, c}(\omega ; p)-E_{\mu, \alpha+1, l}^{\gamma, \delta, k, c}(\omega ; p)\right| \\
& \quad \leq\left(1+\frac{1}{m}\right) E_{\mu, \alpha, l}^{\gamma, \delta, k, c}(\omega ; p) \int_{0}^{1} h(z) d z .
\end{aligned}
$$

Proof In (4.11), putting $a=0$ and $b=1$, then inequality (4.15) is obtained.

Corollary 9 If $m=1$ and $h(z)=z$ in (4.15), then we have

$$
\left|2 E_{\mu, \alpha+2, l}^{\gamma, \delta, k}(\omega ; p)-E_{\mu, \alpha+1, l}^{\gamma, \delta, k, c}(\omega ; p)\right| \leq E_{\mu, \alpha, l}^{\gamma, \delta, k, c}(\omega ; p) .
$$

By applying Theorem 3 similar relations can be established; we leave these for the reader.

\section{Fractional differential equations involving extended generalized Mittag-Leffler function}

In this section, generalized fractional differential equations are solved. The RiemannLiouville fractional derivative operator $D_{a^{+}}^{v}$ is defined as follows:

$$
\left(D_{a^{+}}^{v} f\right)(x)=\left(\frac{d}{d x}\right)^{n}\left(I_{a^{+}}^{n-v} f\right)(x), \quad v \in C ; \Re(v)>0(n=[\Re(v)]+1),
$$

where $\left(I_{a^{+}}^{v} f\right)(x)$ is the Riemann-Liouville fractional integral operator defined as follows:

$$
\left(I_{a^{2}}^{v} f\right)(x)=\frac{1}{\Gamma(v)} \int_{a}^{x}(x-t)^{\nu-1} f(t) d t, \quad x>a .
$$

For $a=0$ the operator $\left(D_{a^{+}}^{v} f\right)(x)$ is represented by $\left(D_{0^{+}}^{v} f\right)(x)$ and $\left(I_{a^{+}}^{v} f\right)(x)$ is represented by $\left(I_{0^{+}}^{v} f\right)(x)$.

The Laplace transform of a function $f(x)$ is defined as follows:

$$
L[f(x) ; s]=\int_{0}^{\infty} e^{-s x} f(x) d x=F(s)
$$


In [34], the Laplace transform of fractional derivative $\left(D_{0^{+}}^{v} f\right)(x)$ is found to be

$$
L\left[D_{0^{+}}^{v} f ; s\right]=s^{\nu} F(s)-\sum_{k=1}^{n} s^{k-1} D_{0^{+}}^{\nu-k} f(0+) \quad(n-1<v<n) \Re(s)>0 .
$$

For more information related to differential equations see Refs. [47-50].

Theorem 10 Let $a \in \mathbb{R}_{+} ; \mu, \alpha, l, \gamma, \nu, c \in C, \Re(\mu), \Re(\alpha), \Re(l)>0, \Re(c)>\Re(\gamma)>0$ with $p \geq$ $0, \delta>0$ and $0<k \leq \delta+\Re(\mu)$. Then, for $x>a$, we have

$$
\begin{aligned}
& D_{a^{+}}^{v}\left[(t-a)^{\alpha-1} E_{\mu, \alpha, l}^{\gamma, \delta, k, c}\left(\omega(t-a)^{\mu} ; p\right)\right](x)=(x-a)^{\alpha-\nu-1} E_{\mu, \alpha-\nu, l}^{\gamma, \delta, k, c}\left(\omega(x-a)^{\mu} ; p\right), \\
& I_{a^{+}}^{v}\left[(t-a)^{\alpha-1} E_{\mu, \alpha, l}^{\gamma, \delta, k, c}\left(\omega(t-a)^{\mu} ; p\right)\right](x)=(x-a)^{\alpha+\nu-1} E_{\mu, \alpha+v, l}^{\gamma, \delta, c}\left(\omega(x-a)^{\mu} ; p\right) .
\end{aligned}
$$

Proof By using the definition of $E_{\mu, \alpha, l}^{\gamma, \delta, k, c}$ defined in (2.2), we have

$$
\begin{aligned}
D_{a^{+}}^{v} & {\left[(t-a)^{\alpha-1} E_{\mu, \alpha, l}^{\gamma, \delta, k, c}\left(\omega(t-a)^{\mu} ; p\right)\right](x) } \\
& =D_{a^{+}}^{v}\left[(t-a)^{\alpha-1} \sum_{n=0}^{\infty} \frac{\beta_{p}(\gamma+n k, c-\gamma)}{\beta(\gamma, c-\gamma)} \frac{(c)_{n k}}{\Gamma(\mu n+\alpha)} \frac{\left(\omega(t-a)^{\mu}\right)^{n}}{(l)_{n \delta}}\right](x) \\
& =\sum_{n=0}^{\infty} \frac{\beta_{p}(\gamma+n k, c-\gamma)}{\beta(\gamma, c-\gamma)} \frac{(c)_{n k}}{\Gamma(\mu n+\alpha)} \frac{\omega^{n}}{(l)_{n \delta}} D_{a^{+}}^{v}\left[(t-a)^{\mu n+\alpha-1}\right](x) .
\end{aligned}
$$

By using the formula $D_{a^{+}}^{v}\left[(t-a)^{\eta}\right](x)=\frac{\Gamma(\eta+1)}{\Gamma(\eta-v+1)}(x-a)^{\eta-v}$, we have

$$
\begin{aligned}
D_{a^{+}}^{v} & {\left[(t-a)^{\alpha-1} E_{\mu, \alpha, l}^{\gamma, \delta, k, c}\left(\omega(t-a)^{\mu} ; p\right)\right](x) } \\
& =\sum_{n=0}^{\infty} \frac{\beta_{p}(\gamma+n k, c-\gamma)}{\beta(\gamma, c-\gamma)} \frac{(c)_{n k}}{\Gamma(\mu n+\alpha-v)} \frac{\left(\omega(x-a)^{\mu}\right)^{n}}{(l)_{n \delta}}(x-a)^{\alpha-\nu-1} \\
& =(x-a)^{\alpha-\nu-1} E_{\mu, \alpha-\nu, l}^{\gamma, \delta, k}\left(\omega(x-a)^{\mu} ; p\right) .
\end{aligned}
$$

Proof of (5.6) is similar to the proof of (5.5) just using the definition of fractional integral operator $I_{a^{+}}^{v}$ therein.

Theorem 11 Let $\mu, \alpha, l, \gamma, v, c \in \mathbb{C}, \Re(\mu), \Re(\alpha), \Re(l)>0, \Re(c)>\Re(\gamma)>0$ with $p \geq 0, \delta>0$ and $0<k \leq \delta+\Re(\mu)$. Then the differential equation

$$
\left(D_{0^{+}}^{v} y\right)(x)=\lambda_{1}\left(\epsilon_{\mu, \alpha, l, \omega, a^{+}}^{\gamma, \delta, k} 1\right)(x ; p)+f(x)
$$

with the initial condition $\left(I_{0^{+}}^{1-\nu}\right)(0+)=C$, has its solution in the space $L(0, \infty)$

$$
\begin{aligned}
y(x)= & C \frac{x^{\nu-1}}{\Gamma(v)}+\lambda_{1} \sum_{n=0}^{\infty} \frac{\beta_{p}(\gamma+n k, c-\gamma)}{\beta(\gamma, c-\gamma)} \frac{(c)_{n k}}{\Gamma(\mu n+\alpha+v+1)} \frac{\omega^{n}}{(l)_{n \delta}} x^{\mu n+\alpha+\nu} \\
& +\frac{1}{\Gamma(\nu)} \int_{0}^{x}(x-t)^{\nu-1} f(t) d t,
\end{aligned}
$$

where $C$ is an arbitrary constant. 
Proof Using the generalized fractional integral operator $\left(\epsilon_{\mu, \alpha, l, \omega, a^{+}}^{\gamma, \delta, k) c}(x ; p)\right.$ given in (2.6) with $a=0$ in (5.9), we have

$$
\left(D_{0^{+}}^{\nu} y\right)(x)=\lambda_{1} x^{\alpha} E_{\mu, \alpha+1, l}^{\gamma, \delta, k, c}\left(\omega x^{\mu} ; p\right)+f(x) .
$$

Applying the Laplace transform on both sides of (5.11), we have

$$
L\left[\left(D_{0^{+}}^{\nu} y\right)(x) ; s\right]=\lambda_{1} L\left[x^{\alpha} E_{\mu, \alpha+1, l}^{\gamma, \delta, k, c}\left(\omega x^{\mu} ; p\right) ; s\right]+L[f(x) ; s] .
$$

First we calculate the Laplace transform of Mittag-Leffler function as follows:

$$
\begin{aligned}
L & {\left[x^{\alpha-1} E_{\mu, \alpha, l}^{\gamma, \delta, k, c}\left(\omega x^{\mu} ; p\right) ; s\right] } \\
& =\int_{0}^{\infty} x^{\alpha} e^{-s x} E_{\mu, \alpha, l}^{\gamma, \delta, k, c}\left(\omega x^{\mu} ; p\right) d x \\
& =\sum_{n=0}^{\infty} \frac{\beta_{p}(\gamma+n k, c-\gamma)}{\beta(\gamma, c-\gamma)} \frac{(c)_{n k}}{\Gamma(\mu n+\alpha)} \frac{\omega^{n}}{(l)_{n \delta}} \int_{0}^{\infty} x^{\alpha+\mu n-1} e^{-s x} d x \\
& =\sum_{n=0}^{\infty} \frac{\beta_{p}(\gamma+n k, c-\gamma)}{\beta(\gamma, c-\gamma)} \frac{(c)_{n k}}{\Gamma(\mu n+\alpha)} \frac{\omega^{n}}{(l)_{n \delta}} \Gamma(\mu n+\alpha) L\left[\frac{x^{\alpha+\mu n-1}}{\Gamma(\mu n+\alpha)} ; s\right] .
\end{aligned}
$$

Since $L\left[\frac{t^{n-1}}{\Gamma(n)}\right]=\frac{1}{s^{n}}(n>0)$, using it in the above, we have

$$
L\left[x^{\alpha-1} E_{\mu, \alpha, l}^{\gamma, \delta, k}\left(\omega x^{\mu} ; p\right) ; s\right]=\frac{1}{s^{\alpha}} \sum_{n=0}^{\infty} \frac{\beta_{p}(\gamma+n k, c-\gamma)}{\beta(\gamma, c-\gamma)} \frac{(c)_{n k}}{(l)_{n \delta}}\left(\frac{\omega}{s^{\mu}}\right)^{n} .
$$

By using (5.3), (5.4) (for $n=1$ ) and (5.14) in (5.12), we have

$$
s^{\nu} y(s)=C+\lambda_{1} s^{-(\alpha+1)} \sum_{n=0}^{\infty} \frac{\beta_{p}(\gamma+n k, c-\gamma)}{\beta(\gamma, c-\gamma)} \frac{(c)_{n k}}{(l)_{n \delta}}\left(\frac{\omega}{s^{\mu}}\right)^{n}+F(s),
$$

which implies that

$$
y(s)=C s^{-v}+\lambda_{1} s^{-(\alpha+v+1)} \sum_{n=0}^{\infty} \frac{\beta_{p}(\gamma+n k, c-\gamma)}{\beta(\gamma, c-\gamma)} \frac{(c)_{n k}}{(l)_{n \delta}}\left(\frac{\omega}{s^{\mu}}\right)^{n}+F(s) s^{-\nu} .
$$

Now taking the inverse Laplace transformation on both sides of (5.15), we have

$$
\begin{aligned}
y(x)= & C \frac{x^{\nu-1}}{\Gamma(\nu)}+\lambda_{1} \sum_{n=0}^{\infty} \frac{\beta_{p}(\gamma+n k, c-\gamma)}{\beta(\gamma, c-\gamma)} \frac{(c)_{n k}}{(l)_{n \delta}} \omega^{n} L^{-1}\left[s^{-(\mu n+\alpha+\nu+1)}\right] \\
& +L^{-1}\left[F(s) s^{-\nu}\right] .
\end{aligned}
$$

After simplification one can get (5.10).

Theorem 12 Let $\mu, \alpha, l, \gamma, \nu, c \in \mathbb{C}, \Re(\mu), \Re(\alpha), \Re(l)>0, \Re(c)>\Re(\gamma)>0$ with $p \geq 0, \delta>0$ and $0<k \leq \delta+\Re(\mu)$. Then the differential equation

$$
\left(D_{0^{+}}^{v} y\right)(x)=\lambda_{1}\left(\epsilon_{\mu, \alpha, l, \omega, a^{+}}^{\gamma, \delta, c}\right)(x ; p)+\lambda_{2} x^{\alpha} E_{\mu, \alpha+1, l}^{\gamma, \delta, k}\left(\omega x^{\mu} ; p\right),
$$


with the initial condition $\left(I_{0^{+}}^{1-\nu}\right)(0+)=C$, has a solution in the space $L(0, \infty)$,

$$
y(x)=C \frac{x^{\nu-1}}{\Gamma(\nu)}+\left(\lambda_{1}+\lambda_{2}\right) \sum_{n=0}^{\infty} \frac{\beta_{p}(\gamma+n k, c-\gamma)}{\beta(\gamma, c-\gamma)} \frac{(c)_{n k}}{\Gamma(\mu n+\alpha+v+1)} \frac{\omega^{n}}{(l)_{n \delta}} x^{\mu n+\alpha+\nu},
$$

where $C$ is an arbitrary constant.

Proof Using the generalized fractional integral operator $\left(\epsilon_{\mu, \alpha, l, \omega, a^{+}}^{\gamma, \delta, k) c}(x ; p)\right.$ given in (2.6) with $a=0$ in (5.16), we have

$$
\begin{aligned}
\left(D_{0^{+}}^{\nu} y\right)(x) & =\lambda_{1} x^{\alpha} E_{\mu, \alpha+1, l}^{\gamma, \delta, k}\left(\omega x^{\mu} ; p\right)+\lambda_{2} x^{\alpha} E_{\mu, \alpha+1, l}^{\gamma, \delta, k, c}\left(\omega x^{\mu} ; p\right) \\
& =\left(\lambda_{1}+\lambda_{2}\right) x^{\alpha} E_{\mu, \alpha+1, l}^{\gamma, \delta, k, c}\left(\omega x^{\mu} ; p\right) .
\end{aligned}
$$

Applying the Laplace transform on both sides of (5.18), we have

$$
L\left[\left(D_{0^{+}}^{\nu} y\right)(x) ; s\right]=\left(\lambda_{1}+\lambda_{2}\right) L\left[x^{\alpha} E_{\mu, \alpha+1, l}^{\gamma, \delta, k, c}\left(\omega x^{\mu} ; p\right) ; s\right] .
$$

By using (5.3), ((5.4) for $n=1)$ and (5.14) in (5.19), we have

$$
s^{v} y(s)=C+\left(\lambda_{1}+\lambda_{2}\right) s^{-(\alpha+1)} \sum_{n=0}^{\infty} \frac{\beta_{p}(\gamma+n k, c-\gamma)}{\beta(\gamma, c-\gamma)} \frac{(c)_{n k}}{(l)_{n \delta}}\left(\frac{\omega}{s^{\mu}}\right)^{n}
$$

which implies that

$$
y(s)=C s^{-\nu}+\left(\lambda_{1}+\lambda_{2}\right) s^{-(\alpha+v+1)} \sum_{n=0}^{\infty} \frac{\beta_{p}(\gamma+n k, c-\gamma)}{\beta(\gamma, c-\gamma)} \frac{(c)_{n k}}{(l)_{n \delta}}\left(\frac{\omega}{s^{\mu}}\right)^{n} .
$$

Now taking the inverse Laplace transformation on both sides of (5.20), we have

$$
y(x)=C \frac{x^{\nu-1}}{\Gamma(\nu)}+\left(\lambda_{1}+\lambda_{2}\right) \sum_{n=0}^{\infty} \frac{\beta_{p}(\gamma+n k, c-\gamma)}{\beta(\gamma, c-\gamma)} \frac{(c)_{n k}}{(l)_{n \delta}} \omega^{n} L^{-1}\left[s^{-(\mu n+\alpha+\nu+1)}\right] .
$$

After simplification one can get (5.17).

Theorem 13 Let $\mu, \alpha, l, \gamma, v, c \in \mathbb{C}, \Re(\mu), \Re(\alpha), \Re(l)>0, \Re(c)>\Re(\gamma)>0$ with $p \geq 0, \delta>0$ and $0<k \leq \delta+\Re(\mu)$. Then the differential equation

$$
\left(D_{0^{+}}^{v} y\right)(x)=\lambda\left(\epsilon_{\mu, \alpha, l, l, \omega, a^{+}}^{\gamma, \delta, k}\right)(x ; p)+\sum_{j=1}^{n}\left[\lambda_{j} x^{\alpha_{j}} E_{\mu_{j}, \alpha_{j}+1, l_{j}}^{\gamma_{j}, \delta_{j}, k_{j}, c_{j}}\left(w_{j} x^{\mu_{j}} ; p\right)\right]
$$

with the initial condition $\left(I_{0^{+}}^{1-v}\right)(0+)=C$, has a solution in the space $L(0, \infty)$,

$$
\begin{aligned}
y(x)= & C \frac{x^{\nu-1}}{\Gamma(v)}+\lambda \sum_{n=0}^{\infty} \frac{\beta_{p}(\gamma+n k, c-\gamma)}{\beta(\gamma, c-\gamma)} \frac{(c)_{n k}}{\Gamma(\mu n+\alpha+v+1)} \frac{\omega^{n}}{(l)_{n \delta}} x^{\mu n+\alpha+v} \\
& +\sum_{j=1}^{n}\left[\lambda_{j} \sum_{n=0}^{\infty} \frac{\beta_{p}\left(\gamma_{j}+n k_{j}, c_{j}-\gamma_{j}\right)}{\beta\left(\gamma_{j}, c_{j}-\gamma_{j}\right)} \frac{\left(c_{j}\right)_{n k_{j}}}{\Gamma\left(\mu_{j} n+\alpha_{j}+v+1\right)} \frac{\omega_{j}^{n}}{\left(l_{j}\right)_{n \delta_{j}}} x^{\mu_{j} n+\alpha_{j}+v}\right],
\end{aligned}
$$

where $C$ is an arbitrary constant. 
Proof Using the generalized fractional integral operator $\left(\epsilon_{\mu, \alpha, l, \omega, a^{+}}^{\gamma, \delta, k) c}(x ; p)\right.$ given in (2.6) with $a=0$ in (5.21), we have

$$
\left(D_{0^{+}}^{\nu} y\right)(x)=\lambda x^{\alpha} E_{\mu, \alpha+1, l}^{\gamma, \delta, k, c}\left(\omega x^{\mu} ; p\right)+\sum_{j=1}^{n}\left[\lambda_{j} x^{\alpha_{j}} E_{\mu_{j}, \alpha_{j}+1, l_{j}}^{\gamma_{j}, \delta_{j}, k_{j}, c_{j}}\left(w_{j} x^{\mu_{j}} ; p\right)\right] .
$$

Applying Laplace transform on both sides of (5.18), we have

$$
\begin{aligned}
L\left[\left(D_{0^{+}}^{\nu} y\right)(x) ; s\right]= & \lambda L\left[x^{\alpha} E_{\mu, \alpha+1, l}^{\gamma, \delta, k, c}\left(\omega x^{\mu} ; p\right) ; s\right] \\
& +L\left[\sum_{j=1}^{n}\left[\lambda_{j} x^{\alpha_{j}} E_{\mu_{j}, \alpha_{j}+1, l_{j}}^{\gamma_{j}, \delta_{j}, k_{j}, c_{j}}\left(w_{j} x^{\mu_{j}} ; p\right)\right] ; s\right] .
\end{aligned}
$$

By using (5.3), ((5.4) for $n=1)$ and (5.14) in (5.24), we have

$$
\begin{aligned}
s^{v} y(s)= & C+\lambda s^{-(\alpha+1)} \sum_{n=0}^{\infty} \frac{\beta_{p}(\gamma+n k, c-\gamma)}{\beta(\gamma, c-\gamma)} \frac{(c)_{n k}}{(l)_{n \delta}}\left(\frac{\omega}{s^{\mu}}\right)^{n} \\
& +\sum_{j=1}^{n}\left[\lambda_{j} s^{-\left(\alpha_{j}+1\right)} \sum_{n=0}^{\infty} \frac{\beta_{p}\left(\gamma_{j}+n k_{j}, c_{j}-\gamma_{j}\right)}{\beta\left(\gamma_{j}, c_{j}-\gamma_{j}\right)} \frac{\left(c_{j}\right)_{n k_{j}}}{\left(l_{j}\right)_{n \delta_{j}}}\left(\frac{\omega_{j}}{s^{\mu_{j}}}\right)^{n}\right],
\end{aligned}
$$

which implies that

$$
\begin{aligned}
y(s)= & C s^{-v}+\lambda s^{-(\alpha+v+1)} \sum_{n=0}^{\infty} \frac{\beta_{p}(\gamma+n k, c-\gamma)}{\beta(\gamma, c-\gamma)} \frac{(c)_{n k}}{(l)_{n \delta}}\left(\frac{\omega}{s^{\mu}}\right)^{n} \\
& +s^{-\nu} \sum_{j=1}^{n}\left[\lambda_{j} s^{-\left(\alpha_{j}+1\right)} \sum_{n=0}^{\infty} \frac{\beta_{p}\left(\gamma_{j}+n k_{j}, c_{j}-\gamma_{j}\right)}{\beta\left(\gamma_{j}, c_{j}-\gamma_{j}\right)} \frac{\left(c_{j}\right)_{n k_{j}}}{\left(l_{j}\right)_{n \delta_{j}}}\left(\frac{\omega_{j}}{s^{\mu_{j}}}\right)^{n}\right] .
\end{aligned}
$$

Now taking the inverse Laplace transformation on both sides of (5.25), we have

$$
\begin{aligned}
y(x)= & C \frac{x^{\nu-1}}{\Gamma(v)}+\lambda \sum_{n=0}^{\infty} \frac{\beta_{p}(\gamma+n k, c-\gamma)}{\beta(\gamma, c-\gamma)} \frac{(c)_{n k}}{(l)_{n \delta}} \omega^{n} L^{-1}\left[s^{-(\mu n+\alpha+v+1)}\right] \\
& +\sum_{j=1}^{n}\left[\lambda_{j} \sum_{n=0}^{\infty} \frac{\beta_{p}\left(\gamma_{j}+n k_{j}, c_{j}-\gamma_{j}\right)}{\beta\left(\gamma_{j}, c_{j}-\gamma_{j}\right)} \frac{\left(c_{j}\right)_{n k_{j}}}{\left(l_{j}\right)_{n \delta_{j}}} \omega_{j} L^{-1}\left[s^{-\left(\mu_{j} n+\alpha_{j}+v+1\right)}\right]\right] .
\end{aligned}
$$

After simplification one can get (5.22).

\section{Concluding remarks}

This research computes the bounds of fractional integral operators containing an extended generalized Mittag-Leffler function in their kernel. These results provide compact formulas for bounds of several kinds of fractional integral operators via several kinds of convexity. By setting specific values to parameters involved in the Mittag-Lefller function some interesting results can be obtained. For example estimations of the fractional integral operators: by Salim and Faraj defined in [39] setting $p=0$, by Rahman et al. defined in [37] by setting $l=\delta=1$, by Shukla and Prajapati defined in [41] by setting $p=0$ and $l=\delta=1$ (see also [42]), by Prabhakar defined in [36] by setting $p=0$ and $l=\delta=k=1$. Also 
inequalities for recurrence relations of Mittag-Leffler functions are obtained via a particular convex function $x^{2}$. At the end some generalized fractional differential equations are solved.

\section{Acknowledgements}

We thank the editor and referees for their careful reading and valuable suggestions to make the article friendly readable. The research work of Zhihua Chen is supported by the Chinese National Natural Science Foundation under Grant 61876047. The research work of Ghulam Farid is supported by the Higher Education Commission of Pakistan under NRPU 2016, Project No. 5421

\section{Funding}

Not applicable.

\section{Availability of data and materials}

There is no additional data required for the finding of results of this paper.

\section{Competing interests}

It is declared that the authors have no competing interests.

\section{Authors' contributions}

All authors have equal contribution in this article. All authors read and approved the final manuscript.

\section{Author details}

${ }^{1}$ Institute of Computing Science and Technology, Guangzhou University, Guangzhou, China. ${ }^{2}$ Department of Mathematics, COMSATS University Islamabad, Attock, Pakistan. ${ }^{3}$ General Studies Department, Jubail Industrial College, Jubail Industrial City, Jubail, Kingdom of Saudi Arabia.

\section{Publisher's Note}

Springer Nature remains neutral with regard to jurisdictional claims in published maps and institutional affiliations.

Received: 18 November 2019 Accepted: 7 April 2020 Published online: 17 April 2020

\section{References}

1. Agarwal, R.P.: A propos d'une note de m. Pierre Humbert. C. R. Math. Acad. Sci. 236, 2031-2032 (1953)

2. Andrić, M., Farid, G., Pečarić, J.: A further extension of Mittag-Leffler function. Fract. Calc. Appl. Anal. 21, 1377-1395 (2018)

3. Aydogan, M.S., Baleanu, D., Mousalou, A., Rezapour, S.: On high order fractional integro-differential equations including the Caputo-Fabrizio derivative. Bound. Value Probl. 2018, Article ID 90 (2018)

4. Bagley, R.L., Torvik, P.: A theoretical basis for the application of fractional calculus to viscoelasticity. J. Rheol. 27, 201-210 (1983)

5. Baleanu, D., Asad, J.H., Jajarmi, A.: New aspects of the motion of a particle in a circular cavity. Proc. Rom. Acad., Ser. A: Math. Phys. Tech. Sci. Inf. Sci. 19(2), 361-367 (2018)

6. Baleanu, D., Etemad, S., Pourrazi, S., Rezapour, S.: On the new fractional hybrid boundary value problems with three-point integral hybrid conditions. Adv. Differ. Equ. 2019, Article ID 473 (2019)

7. Baleanu, D., Ghafarnezhad, K., Rezapour, S., Shabibi, M.: On the existence of solutions of a three steps crisis integro-differential equation. Adv. Differ. Equ. 2018, Article ID 135 (2018)

8. Baleanu, D., Jajarmi, A., Hajipour, M.: A new formulation of the fractional optimal control problems involving Mittag-Leffler nonsingular kernel. J. Optim. Theory Appl. 175, 718-737 (2017)

9. Baleanu, D., Jajarmi, A., Sajjadi, S.S., Mozyrska, D.: A new fractional model and optimal control of a tumor-immune surveillance with non-singular derivative operator. Chaos 29, Article ID 083127 (2019)

10. Baleanu, D., Rezapour, S., Saberpour, Z.: On fractional integro-differential inclusions via the extended fractional Caputo-Fabrizio derivation. Bound. Value Probl. 2019, Article ID 79 (2019)

11. Baleanu, D., Sajjadi, S.S., Jajarmi, A., Asad, J.H.: New features of the fractional Euler-Lagrange equations for a physical system within non-singular derivative operator. Eur. Phys. J. Plus 134, Article ID 181 (2019)

12. Doungmo Goufo, E.F., Mugisha, S.: On analysis of fractional Navier-Stokes equations via nonsingular solutions and approximation. Math. Probl. Eng. 2015, Article ID 212760 (2015)

13. Dragomir, S.S., Agarawal, R.P.: Two inequalities for differentiable mappings and applications to special means of real numbers and to trapezoidal formula. Appl. Math. Lett. 11,91-95 (1980)

14. Farid, G.: Some Riemann-Liouville fractional integral inequalities for convex functions. J. Anal. 27, 1095-1102 (2019)

15. Farid, G.: Bounds of Riemann-Liouville fractional integral operators. Comput. Methods Differ. Equ. (in press)

16. Farid, G., Khan, K.A., Latif, N., Rehman, A.U., Mehmood, S.: General fractional integral inequalities for convex and m-convex functions via an extended generalized Mittag-Leffler function. J. Inequal. Appl. 2018, Article ID 243 (2018)

17. Farid, G., Rehman, A.U., Ullah, S., Nosheen, A., Waseem, M., Mehboob, Y.: Opial-type inequalities for convex function and associated results in fractional calculus. Adv. Differ. Equ. 2019, Article ID 152 (2019)

18. Fujioka, J., Espinosa, A., Rodríguez, R.F., Malomed, B.A.: Radiating subdispersive fractional optical solitons. Chaos 24 Article ID 033121 (2014)

19. Hajipour, M., Jajarmi, A., Baleanu, D., Sun, H.G.: On an accurate discretization of a variable-order fractional reaction-diffusion equation. Commun. Nonlinear Sci. Numer. Simul. 69, 119-133 (2019) 
20. Haubold, H.J., Mathai, A.M., Saxena, R.K.: Mittag-Leffler functions and their applications. J. Appl. Math. 2011, Article ID 298628 (2011)

21. Hilfer, R.: Applications of Fractional Calculus in Physics. World Scientific, Singapore (2000)

22. Ionescu, C., Desager, K., Keyser, R.D.: Fractional order model parameters for the respiratory input impedance in healthy and in asthmatic children. Comput. Methods Programs Biomed. 101, 315-323 (2011)

23. Ionescu, C.M., Keyser, R.D.: Relations between fractional-order model parameters and lung pathology in chronic obstructive pulmonary disease. IEEE Trans. Biomed. Eng. 56, 978-987 (2009)

24. Jajarmi, A., Arshad, S., Baleanu, D.: A new fractional modelling and control strategy for the outbreak of dengue fever. Phys. A, Stat. Mech. Appl. 535, Article ID 122524 (2019)

25. Jajarmi, A., Ghanbari, B., Baleanu, D.: A new and efficient numerical method for the fractional modeling and optimal control of diabetes and tuberculosis co-existence. Chaos 29, Article ID 093111 (2019)

26. Jajarmi, A., Hajipour, M., Mohammadzadeh, E., Baleanu, D.: A new approach for the nonlinear fractional optimal control problems with external persistent disturbances. J. Franklin Inst. 335, 3938-3967 (2018)

27. Kilbas, A.A., Saigo, M., Saxena, R.K.: Generalized Mittag-Leffler function and generalized fractional calculus operators. Integral Transforms Spec. Funct. 15, 31-49 (2004)

28. Kwuimy, C.K., Litak, G., Nataraj, C.: Nonlinear analysis of energy harvesting systems with fractional order physical properties. Nonlinear Dyn. 80, 491-501 (2015)

29. Magin, R.L.: Fractional Calculus in Bioengineering. Begell House, Redding (2006)

30. Mehmood, S., Farid, G.: Fractional integrals inequalities for exponentially $m$-convex functions. Open J. Math. Sci. 4 $78-85(2020)$

31. Mehmood, S., Farid, G., Khan, K.A., Yussouf, M.: New fractional Hadamard and Fejér-Hadamard inequalities associated with exponentially $(h, m)$-convex functions. Eng. Appl. Sci. Lett. 3, 9-18 (2020)

32. Mehmood, S., Farid, G., Khan, K.A., Yussouf, M.: New Hadamard and Fejér-Hadamard fractional inequalities for exponentially m-convex function. Eng. Appl. Sci. Lett. 3, 45-55 (2020)

33. Mittag-Leffler, G.: Sur la nouvelle fonction $E_{\alpha}(x)$. C. R. Math. Acad. Sci. 137, 554-558 (1903)

34. Oldham, K.B., Spanier, J.: The Fractional Calculus: Theory and Applications of Differentiation and Integration to Arbitrary Order. Academic Press, New York (1974)

35. Özdemir, M.E., Akdemri, A.O., Set, E.: On $(h-m)$-convexity and Hadamard-type inequalities. Transylv. J. Math. Mech. 8, 51-58 (2016)

36. Prabhakar, T.R.: A singular integral equation with a generalized Mittag-Leffler function in the kernel. Yokohama Math. J. 19, 7-15 (1971)

37. Rahman, G., Baleanu, D., Qurashi, M.A., Purohit, S.D., Mubeen, S., Arshad, M.: The extended Mittag-Leffler function via fractional calculus. J. Nonlinear Sci. Appl. 10, 4244-4253 (2017)

38. Salim, T.O.: Some properties relating to the generalized Mittag-Leffler function. Adv. Appl. Math. Anal. 4, 21-30 (2009)

39. Salim, T.O., Faraj, A.W.: A generalization of Mittag-Leffler function and integral operator associated with integral calculus. J. Fract. Calc. Appl. 3, 1-13 (2012)

40. Saxena, R.K., Mathai, A.M., Haubold, H.J.: On fractional kinetic equations. Astrophys. Space Sci. 282, $281-287$ (2002)

41. Shukla, A.K., Prajapati, J.C.: On a generalization of Mittag-Leffler function and its properties. J. Math. Anal. Appl. 336, 797-811 (2007)

42. Srivastava, H.M., Tomovski, Z.: Fractional calculus with an integral operator containing generalized Mittag-Leffler function in the kernel. Appl. Math. Comput. 211, 198-210 (2009)

43. Tomovski, Z., Hiller, R., Srivastava, H.M.: Fractional and operational calculus with generalized fractional derivative operators and Mittag-Leffler function. Integral Transforms Spec. Funct. 21, 797-814 (2011)

44. Ullah, S., Farid, G., Khan, K.A., Waheed, A., Mehmood, S.: Generalized fractional inequalities for quasi-convex functions. Adv. Differ. Equ. 2019, Article ID 15 (2019)

45. Varosanec, S.: On h-convexity. J. Math. Anal. Appl. 326, 303-311 (2007)

46. Wiman, A.: Uber den fundamental satz in der theori der functionen. Acta Math. 29, 191-201 (1905)

47. Yang, A.M., Han, Y., Li, J., Liu, W.X.: On steady heat flow problem involving Yang-Srivastava-Machado fractional derivative without singular kernel. Therm. Sci. 20, 717-721 (2016)

48. Yang, X.J., Gao, F., Ju, Y., Zhou, H.W.: Fundamental solutions of the general fractional-order diffusion equations. Math Methods Appl. Sci. 41, 9312-9320 (2018)

49. Yang, X.J., Machado, J.A.T., Baleanu, D.: Anomalous diffusion models with general fractional derivatives within the kernels of the extended Mittag-Leffler type functions. Rom. Rep. Phys. 69, Article ID 20 (2017)

50. Yang, X.J., Srivastava, H.M., Machado, J.A.T.: A new fractional derivative without singular kernel application to the modeling of the steady heat flow. Therm. Sci. 20,753-756 (2016)

51. Yusuf, A., Inc, M., Aliyu, A., Baleanu, D.: Optical solitons possessing beta derivative of the Chen-Lee-Liu equation in optical fiber. Front. Phys. 7, Article ID 34 (2019)

52. Zhao, D., Farid, G., Zeb, M., Ahmad, S., Mahreen, K.: On boundedness of unified integral for quasiconvex functions. Adv. Differ. Equ. 2020, Article ID 38 (2020) 